\title{
Off-shell production of top-antitop pairs in the lepton+jets channel at NLO QCD
}

\author{
Ansgar Denner and Mathieu Pellen \\ Universität Würzburg, Institut für Theoretische Physik und Astrophysik, \\ 97074 Würzburg, Germany \\ E-mail: ansgar.denner@physik.uni-wuerzburg.de, \\ mathieu.pellen@physik.uni-wuerzburg.de
}

ABSTRACT: The production of top-quark pairs that subsequently decay hadronically and leptonically (lepton+jets channel) is one of the key processes for the study of top-quark properties at the LHC. In this article, NLO QCD corrections of order $\mathcal{O}\left(\alpha_{\mathrm{s}}^{3} \alpha^{4}\right)$ to the hadronic process pp $\rightarrow \mu^{-} \bar{\nu}_{\mu} \mathrm{b} \overline{b j j}$ are presented. The computation includes off-shell as well as non-resonant contributions, and experimental event selections are used in order to provide realistic predictions. The results are provided in the form of cross sections and differential distributions. The QCD corrections are sizeable and different from the ones of the fully leptonic channel. This is due to the different final state where here four jets are present at leading order.

KEYWORDS: NLO Computations

ArXiv EPrint: 1711.10359 


\section{Contents}

1 Introduction 1

2 Definition of the process 2

3 Details of the calculation 4

4 Numerical analysis $\quad 7$

$\begin{array}{lll}4.1 & \text { Input parameters } & 7\end{array}$

4.2 Event selection 8

4.3 Cross sections 9

4.4 Differential distributions 11

$\begin{array}{llr}5 & \text { Conclusion } & 17\end{array}$

\section{Introduction}

The large amount of data collected during the run II of the Large Hadron Collider (LHC) will allow to probe the high-energy behaviour of many Standard Model processes. This is particularly important as the high-energy tails of differential distributions are expected to be sensitive to new-physics contributions. In order to achieve stringent tests of the Standard Model, theoretical predictions should be computed with at least next-to-leading order (NLO) QCD and electroweak (EW) accuracy. In addition, in order to be directly comparable with experiments, the calculations should be differential in the final states that are actually measured in experiments. The last point is particularly crucial as in the highenergy tails of differential distributions, off-shell and non-resonant contributions become increasingly relevant. Thus, theoretical predictions should include as much as possible offshell as well as non-resonant effects in order to describe appropriately the final states seen experimentally.

In this regard, the production of top-antitop pairs is exemplary. In the past few years, several off-shell computations have been performed for this process. First, NLO QCD corrections [1-5] have been calculated and matched to parton shower in the narrow-width approximation [6] and recently accounting for the resonance structure of the process [7]. The NLO EW corrections have been computed recently [8]. In addition, the results for the off-shell production of a top-antitop pair in association with a jet at NLO QCD $[9,10]$ or in association with a Higgs boson at NLO QCD and EW [11, 12] are available. Recently, an approximate NNLO QCD computation including decays [13] was published. This seems to reproduce well the full NNLO QCD results $[14,15]$ for on-shell top-quark production but does not account for non-resonant top-quark contributions that can be significant [16-18].

For now, all these computations have focused on the channel where both top quarks decay leptonically. From the theoretical point of view, this channel is preferred as it 
contains only two strongly interacting particles in the final state (two bottom quarks). However, experimentally, the channel where one top quark decays hadronically (denoted hadronic top quark) while the other decays leptonically (denoted leptonic top quark) is also investigated $[19,20]$. It is dubbed lepton+jets channel, features a larger cross section, and has the advantage to allow for a better reconstruction of the event as only one neutrino contributes to the missing transverse energy (as opposed to the fully leptonic channel where two neutrinos carry away some momentum).

For this reason, we have computed for the first time the NLO QCD corrections to the production of top-antitop pairs in the lepton+jets channel, i.e. the process $\mathrm{pp} \rightarrow \mu^{-} \bar{\nu}_{\mu} \mathrm{b} \bar{b} j \mathrm{j}$. We have considered the order $\mathcal{O}\left(\alpha_{\mathrm{s}}^{2} \alpha^{4}\right)$ contributions at leading order (LO) and present the NLO QCD corrections at the order $\mathcal{O}\left(\alpha_{\mathrm{s}}^{3} \alpha^{4}\right)$. The computation features all off-shell and non-resonant effects to the partonic channels that involve two resonant top quarks. In particular, it allows for a direct comparison with experimental measurements as the event selection applied to the final state follows the experimental one. The corrections are sizeable and different from the ones to the top-pair-production process with two leptonically decaying top quarks. In particular, they can be much larger in some phase-space regions. This originates from the different final state where here four jets are present at LO (two light jets and two bottom jets). In particular, the increased number of jets in the final state and the corresponding irreducible background can alter the predictions significantly. This is discussed in detail at the level of the fiducial cross section and in several differential distributions. More precisely, new effects show up in the tails of the transverse momentum distributions as well as in other regions that are sizeably affected by non-resonant contributions.

In this article we are focusing on the NLO QCD corrections to the production of a pair of top quarks and the corresponding off-shell effects. We do not include suppressed contributions such as partonic channels that do not involve two resonant top quarks and interferences of amplitudes of order $\mathcal{O}\left(g_{\mathrm{s}}^{4} g^{2}\right)$ with those of order $\mathcal{O}\left(g^{6}\right)$. Furthermore, we do not take into account bottom-quark-induced and photon-induced contributions, which are suppressed owing to the involved parton distributions functions (PDFs). The corresponding LO contributions are at the per-mille level of the fiducial cross section for top-pair production and thus negligible with respect to the experimental precision at the LHC. Since all these suppressed contributions are of the order of the numerical accuracy of our NLO predictions and not visible in the presented results for distributions, we decided not to include them.

The article is organised as follows: in section 2 the process studied is defined, while in section 3 the technical details of the calculation are presented. Section 4 is devoted to the numerical results and their discussion. In particular, cross sections as well as differential distributions are presented. Finally, section 5 contains a summary and concluding remarks.

\section{Definition of the process}

We consider the off-shell production of top-antitop pairs in the lepton+jets channel at the LHC, i.e. the hadronic process

$$
\mathrm{pp} \rightarrow \mu^{-} \bar{\nu}_{\mu} \mathrm{b} \overline{b j j}
$$


up to 2 resonant top quarks up to 2 resonant $W$ bosons no resonant top quark one resonant $\mathrm{W}$ boson

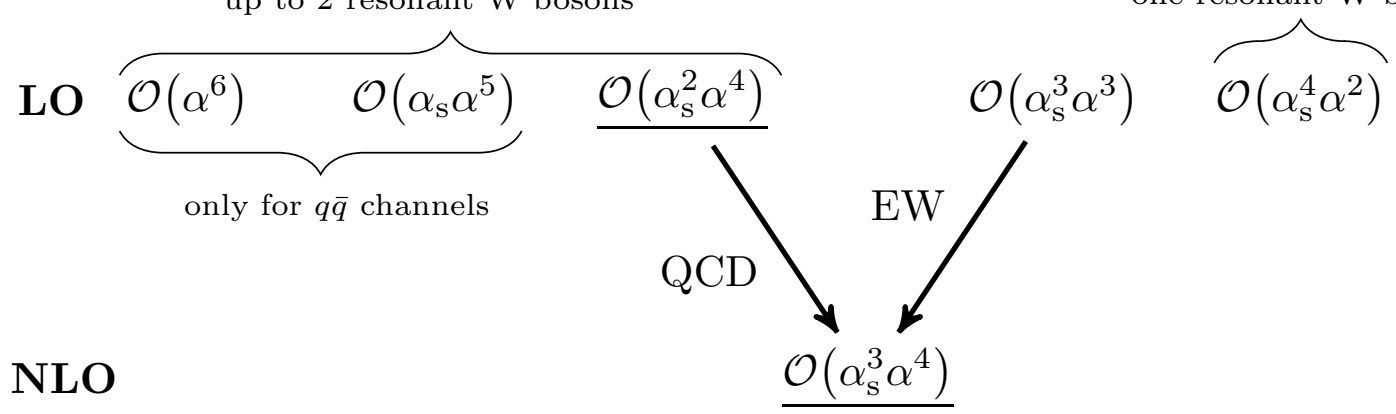

Figure 1. Graphical representation of contributions to the cross section of pp $\rightarrow \mu^{-} \bar{\nu}_{\mu} \mathrm{b} \bar{b} \mathrm{jj}$. At NLO, the order $\mathcal{O}\left(\alpha_{\mathrm{s}}^{3} \alpha^{4}\right)$ receives QCD corrections and EW correction to the orders $\mathcal{O}\left(\alpha_{\mathrm{s}}^{2} \alpha^{4}\right)$ and $\mathcal{O}\left(\alpha_{\mathrm{s}}^{3} \alpha^{3}\right)$, respectively. The two underlined contributions $\left[\mathcal{O}\left(\alpha_{\mathrm{s}}^{2} \alpha^{4}\right)\right.$ at LO and $\mathcal{O}\left(\alpha_{\mathrm{s}}^{3} \alpha^{4}\right)$ at NLO] are the ones considered in the present calculation.
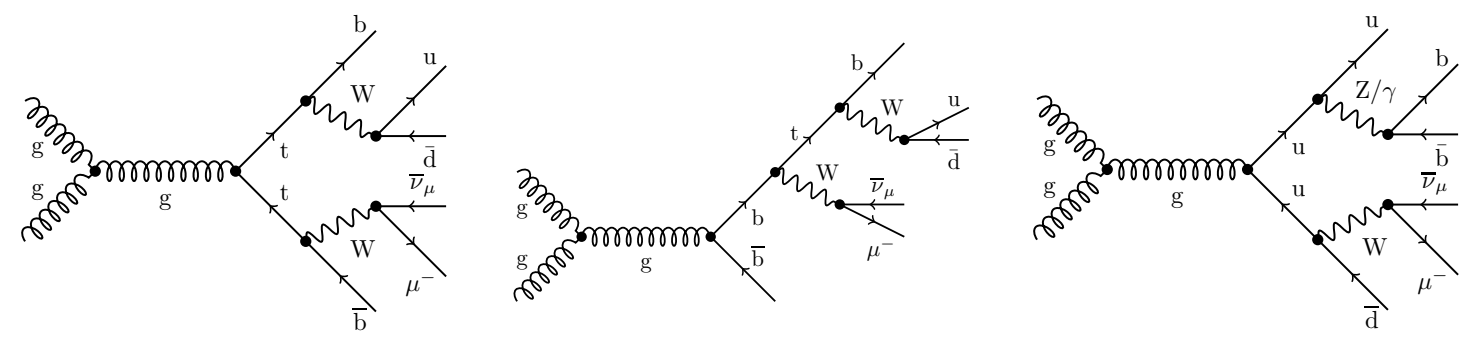

Figure 2. Sample tree-level Feynman diagrams of order $\mathcal{O}\left(g_{\mathrm{s}}^{2} g^{4}\right)$ for gg $\rightarrow \mu^{-} \bar{\nu}_{\mu}$ bbjjj. Some diagrams have two resonant top quarks and two resonant $\mathrm{W}$ bosons (left) while some have only one resonant top quark and two resonant $\mathrm{W}$ bosons (middle) or no resonant top quark and one resonant W boson (right).

At the matrix-element level, this process possesses three types of LO contributions of orders $\mathcal{O}\left(g^{6}\right), \mathcal{O}\left(g_{\mathrm{s}}^{2} g^{4}\right)$, and $\mathcal{O}\left(g_{\mathrm{s}}^{4} g^{2}\right)$. The corresponding contributions at the cross-section level are shown in figure 1. Among these, the dominant one is of order $\mathcal{O}\left(\alpha_{\mathrm{s}}^{2} \alpha^{4}\right)$. Sample diagrams contributing at the order $\mathcal{O}\left(g_{\mathrm{s}}^{2} g^{4}\right)$ are displayed in figure 2. There are contributions involving two resonant top quarks and two resonant $\mathrm{W}$ bosons (left), contributions with one resonant top quark and two resonant $\mathrm{W}$ bosons (middle), and contributions with no resonant top quark and one resonant $\mathrm{W}$ boson (right). The two contributions of orders $\mathcal{O}\left(\alpha^{6}\right)$ and $\mathcal{O}\left(\alpha_{\mathrm{S}} \alpha^{5}\right)$ are suppressed owing to power counting in the two coupling constants and because they exist only for $q \bar{q}$ channels (which is suppressed with respect to the gg channel at the LHC). The contributions of orders $\mathcal{O}\left(\alpha_{\mathrm{s}}^{3} \alpha^{3}\right)$ and $\mathcal{O}\left(\alpha_{\mathrm{s}}^{4} \alpha^{2}\right)$ are suppressed due to the absence of doubly resonant top quarks/W bosons. The NLO QCD corrections to the dominant contribution are thus of order $\mathcal{O}\left(\alpha_{\mathrm{s}}^{3} \alpha^{4}\right)$. As described in figure 1, these NLO corrections consist of QCD and EW corrections to the orders $\mathcal{O}\left(\alpha_{\mathrm{s}}^{2} \alpha^{4}\right)$ and $\mathcal{O}\left(\alpha_{\mathrm{s}}^{3} \alpha^{3}\right)$, respectively. Contributions at order $\mathcal{O}\left(\alpha_{\mathrm{s}}^{2} \alpha^{4}\right)$ also arise from the interference of amplitudes 
of orders $\mathcal{O}\left(g_{\mathrm{s}}^{4} g^{2}\right)$ and $\mathcal{O}\left(g^{6}\right)$. These contributions are strongly suppressed, since they only arise in $q \bar{q}$ channels and the $\mathcal{O}\left(g_{\mathrm{s}}^{4} g^{2}\right)$ amplitude does not involve resonant top quarks and only one resonant $\mathrm{W}$ boson. The corresponding LO contributions are at the level of $10^{-6}$ for the fiducial cross section and therefore completely negligible.

Using symmetries between different quark families, the fully leptonic process can be built from only four independent partonic processes: the ones with initial states gg, uu $/ \bar{u} u$, $\mathrm{d} \overline{\mathrm{d}} / \overline{\mathrm{d}} \mathrm{d}$, and $\mathrm{b} \overline{\mathrm{b}} / \overline{\mathrm{b}} \mathrm{b}$. For the semi-hadronic decay of the top-quark pair, the number of independent partonic channels rises to 32. Among these, the six partonic channels that feature two resonant top quarks approximate the LO fiducial cross section at the level of per mille $(0.28 \%)$ for the set-up described in sections 4.1 and 4.2 . The other channels, which can be constructed upon crossing one or two final state quarks in the initial state involve at most one top-quark and one $\mathrm{W}$-boson resonance. They are further suppressed by a di-jet invariant-mass cut. Relaxing this cut, these contributions become of the order of a couple of per cent $(2.0 \%)$ of the fiducial cross section at LO. The channels involving two resonant top quarks read:

$$
\begin{aligned}
\mathrm{gg} & \rightarrow \mu^{-} \bar{\nu}_{\mu} \mathrm{b} \overline{\mathrm{b}} q_{i} \bar{q}_{j}, & q_{i} q_{j} & \in\{\mathrm{ud}, \mathrm{cs}\}, \\
q_{i} \bar{q}_{i} / \bar{q}_{i} q_{i} & \rightarrow \mu^{-} \bar{\nu}_{\mu} \mathrm{b} \overline{\mathrm{b}} q_{i} \bar{q}_{j}, & q_{i} q_{j} & \in\{\mathrm{ud}, \mathrm{cs}\}, \\
q_{i} \bar{q}_{i} / \bar{q}_{i} q_{i} & \rightarrow \mu^{-} \bar{\nu}_{\mu} \mathrm{b} \overline{\mathrm{b}} q_{j} \bar{q}_{k}, & q_{i} q_{j} q_{k} & \in\{\mathrm{ucs}, \mathrm{cud}\}, \\
q_{i} \bar{q}_{i} / \bar{q}_{i} q_{i} & \rightarrow \mu^{-} \bar{\nu}_{\mu} \mathrm{b} \overline{\mathrm{b}} q_{j} \bar{q}_{i}, & q_{i} q_{j} & \in\{\mathrm{du}, \mathrm{sc}\}, \\
q_{i} \bar{q}_{i} / \bar{q}_{i} q_{i} & \rightarrow \mu^{-} \bar{\nu}_{\mu} \mathrm{b} \overline{\mathrm{b}} q_{j} \bar{q}_{k}, & q_{i} q_{j} q_{k} & \in\{\mathrm{dcs}, \mathrm{sud}\}, \\
\mathrm{b} \overline{\mathrm{b}} / \overline{\mathrm{b}} \mathrm{b} & \rightarrow \mu^{-} \bar{\nu}_{\mu} \mathrm{b} \overline{\mathrm{b}} q_{i} \bar{q}_{j}, & q_{i} q_{j} & \in\{\mathrm{ud}, \mathrm{cs}\} .
\end{aligned}
$$

For the fiducial cross section considered in this work, the LO bottom-quark contributions turn out to be $0.13 \%$. Since this is below the integration error of the NLO calculation $(0.5 \%)$ we do not include them in the cross sections and differential distributions presented in this article. Therefore, in the following computation, only the five remaining partonic channels and the corresponding NLO QCD corrections are considered.

In addition there are contributions from photon-induced channels, which are, however, suppressed by the photon PDFs. The leading photon-induced contribution arises from the process $\mathrm{g} \gamma / \gamma \mathrm{g} \rightarrow \mu^{-} \bar{\nu}_{\mu} \mathrm{b} \overline{\mathrm{b}} q_{l} \bar{q}_{m}$ at order $\mathcal{O}\left(\alpha_{\mathrm{S}} \alpha^{5}\right)$. It is enhanced owing to the gluon PDF and the fact that it possesses doubly-resonant top-quark contributions, but it is suppressed by a factor $\alpha / \alpha_{\mathrm{S}}$ with respect to the leading gg- and $q \bar{q}$-induced contributions. At the order $\mathcal{O}\left(\alpha_{\mathrm{s}} \alpha^{5}\right)$, it amounts to $0.31 \%$ of the LO fiducial cross section. These findings are in line with the ones of refs. $[8,12]$ given that the present number has been obtained with the LUXqed_plus_PDF4LHC15_nnlo_100 set [21]. All other photon-induced contributions at LO or NLO can only be a fraction of these because of PDF suppression, coupling suppression, and/or lacking resonance enhancement.

\section{Details of the calculation}

The Monte Carlo program used for this computation has already been employed for NLO computations involving off-shell top quarks $[8,11,12]$. In addition, this program has also 

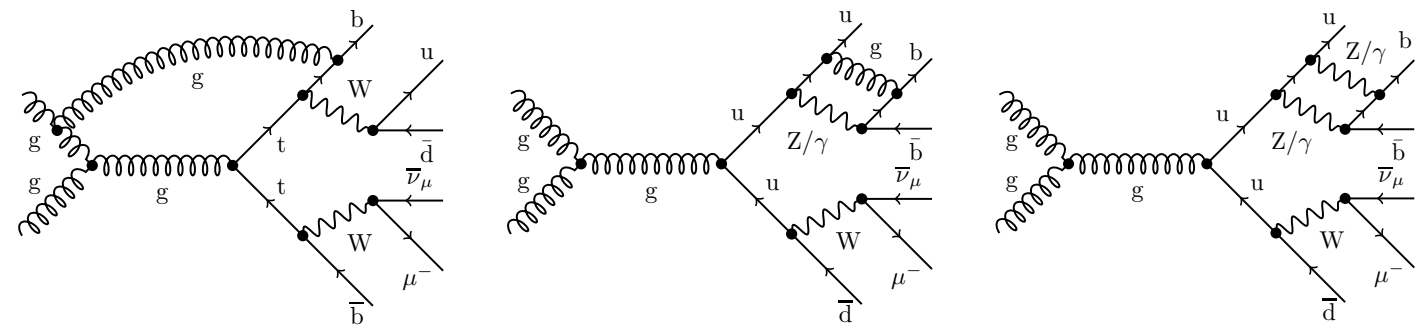

Figure 3. Sample one-loop Feynman diagrams contributing to gg $\rightarrow \mu^{-} \bar{\nu}_{\mu}$ b $\overline{b j j}$ at order $\mathcal{O}\left(\alpha_{\mathrm{s}}^{3} \alpha^{4}\right)$ at NLO. While some diagrams can be uniquely identified as QCD corrections (left) or EW corrections (right), this is not possible for others (middle).

been used for the NLO QCD and EW computation of the process pp $\rightarrow \mu^{+} \nu_{\mu} \mathrm{e}^{+} \nu_{\mathrm{ejj}}$ [22] where also two QCD jets are present at LO. Finally, the program uses similar phase-space mappings to those of refs. [23-25] to ensure a fast integration even for processes with high multiplicities.

Virtual corrections: we include virtual corrections obtained from all one-loop amplitudes interfered with tree amplitudes giving rise to an order $\mathcal{O}\left(\alpha_{\mathrm{s}}^{3} \alpha^{4}\right)$ contribution for all the processes described in eq. (2.2). This includes, in particular, one-loop amplitudes of order $\mathcal{O}\left(g_{s}^{4} g^{4}\right)$ interfered with $\mathcal{O}\left(g_{s}^{2} g^{4}\right)$ tree amplitudes. Such loop amplitudes are obtained upon inserting a gluon into the tree-level diagrams of order $\mathcal{O}\left(g_{s}^{2} g^{4}\right)$ for the processes of eq. (2.2). Examples are shown in the left and the middle of figure 3.

Virtual corrections of order $\mathcal{O}\left(\alpha_{\mathrm{s}}^{3} \alpha^{4}\right)$ can also been obtained by interfering one-loop amplitudes of order $\mathcal{O}\left(g_{s}^{2} g^{6}\right)$ [which would usually be referred to as EW corrections to the dominating LO diagrams of order $\left.\mathcal{O}\left(g_{s}^{2} g^{4}\right)\right]$ with $\mathcal{O}\left(g_{s}^{4} g^{2}\right)$ tree-level amplitudes. Such an EW one-loop diagram is depicted on the right-hand side of figure 3, while a tree-level diagram of order $\mathcal{O}\left(g_{s}^{4} g^{2}\right)$ would for instance result from the one in the right-hand side of figure 2 upon replacing the $\mathrm{Z}$ boson or photon by a gluon. LO contributions of order $\mathcal{O}\left(g_{s}^{4} g^{2}\right)$ exist only for the process pp $\rightarrow \mu^{-} \bar{\nu}_{\mu} \mathrm{b} \overline{b j j}$ but not for the $\mu^{-} \bar{\nu}_{\mu} \mathrm{b} \overline{\mathrm{be}}{ }^{+} \nu_{\mathrm{e}}$ final state relevant for two leptonically decaying top quarks. The situation is similar to the case of NLO EW corrections to the fully leptonic process where interferences with one-loop QCD corrections have to be considered [8]. We note that these contributions are numerically small as they feature only one resonant $\mathrm{W}$ boson but they must be included in order to ensure an infrared (IR) finite result.

Finally virtual corrections of order $\mathcal{O}\left(\alpha_{\mathrm{s}}^{3} \alpha^{4}\right)$ also result upon interfering one-loop amplitudes of order $\mathcal{O}\left(g_{s}^{6} g^{2}\right)$, i.e. QCD corrections to the suppressed LO diagrams of order $\mathcal{O}\left(g_{s}^{4} g^{2}\right)$, with $\mathcal{O}\left(g^{6}\right)$ tree-level amplitudes. These corrections can be separated on the basis of Feynman diagrams and have been neglected. Since such contributions can be uniquely identified as QCD corrections, the associated IR singularities cancel upon adding the corresponding real-radiation contributions. Numerically, they are well below the per-mille level of the LO cross section and are thus irrelevant. 
All the tree-level and one-loop matrix elements have been obtained from the public code Recola $[26,27] .{ }^{1}$ It uses the Collier $[31,32]$ library to calculate the one-loop scalar [33-36] and tensor integrals [37-39] numerically. The complex-mass scheme $[24,40]$ is used throughout.

Real radiation: the real QCD corrections are obtained by attaching a gluon in all possible ways to the Born processes listed in eq. (2.2). Consequently, one has to consider four types of processes with all possible quark-flavour combinations:

$$
\begin{aligned}
\mathrm{gg} & \rightarrow \mu^{-} \bar{\nu}_{\mu} \mathrm{b} \overline{\mathrm{b}} q_{l} \bar{q}_{m} \mathrm{~g}, \\
q_{i} \bar{q}_{i} / \bar{q}_{i} q_{i} & \rightarrow \mu^{-} \bar{\nu}_{\mu} \mathrm{b} \overline{\mathrm{b}} q_{l} \bar{q}_{m} \mathrm{~g}, \\
q_{i} \mathrm{~g} / \mathrm{g} q_{i} & \rightarrow \mu^{-} \bar{\nu}_{\mu} \mathrm{b} \overline{\mathrm{b}} q_{l} \bar{q}_{m} q_{i}, \\
\bar{q}_{i} \mathrm{~g} / \mathrm{g} \bar{q}_{i} & \rightarrow \mu^{-} \bar{\nu}_{\mu} \mathrm{b} \overline{\mathrm{b}} q_{l} \bar{q}_{m} \bar{q}_{i},
\end{aligned}
$$

with $q_{i} \in\{\mathrm{u}, \mathrm{d}, \mathrm{c}, \mathrm{s}\}$ and $q_{l} q_{m} \in\{\mathrm{ud}, \mathrm{cs}\}$. Moreover, real photon radiation to the interferences of order $\mathcal{O}\left(g_{s}^{2} g^{4}\right)$ and $\mathcal{O}\left(g_{s}^{4} g^{2}\right)$ contributions have to be taken into account in order to ensure IR finiteness of the corrections of order $\mathcal{O}\left(\alpha_{\mathrm{s}}^{3} \alpha^{4}\right)$. Thus, the following processes have to be included:

$$
\begin{aligned}
\mathrm{gg} & \rightarrow \mu^{-} \bar{\nu}_{\mu} \mathrm{b} \bar{b} q_{l} \bar{q}_{m} \gamma, \\
q_{i} \bar{q}_{i} / \bar{q}_{i} q_{i} & \rightarrow \mu^{-} \bar{\nu}_{\mu} \mathrm{b} \overline{\mathrm{b}} q_{l} \bar{q}_{m} \gamma,
\end{aligned}
$$

with $q_{i} \in\{\mathrm{u}, \mathrm{d}, \mathrm{c}, \mathrm{s}\}$ and $q_{l} q_{m} \in\{\mathrm{ud}, \mathrm{cs}\}$.

Note that the photon-induced real corrections of order $\mathcal{O}\left(\alpha_{\mathrm{s}}^{3} \alpha^{4}\right)$ resulting from $q_{i} \gamma / \gamma q_{i} \rightarrow \mu^{-} \bar{\nu}_{\mu} \mathrm{b} \overline{\mathrm{b}} q_{l} \bar{q}_{m} q_{i}$ and $\mathrm{g} \gamma / \gamma q_{i} \rightarrow \mu^{-} \bar{\nu}_{\mu} \mathrm{b} \overline{\mathrm{b}} q_{l} \bar{q}_{m} \mathrm{~g}$ have been neglected. They are suppressed owing to the photon PDF and the fact that in the resulting $\mathcal{O}\left(\alpha_{\mathrm{s}}^{3} \alpha^{4}\right)$ contributions at least one of the amplitudes does not feature doubly-resonant top contributions. They are expected to be smaller than the LO photon-induced contributions discussed at the end of section 2 and therefore negligible.

To handle the IR singularities in the real contributions, the dipole subtraction method [41, 42] for both QCD and QED has been used. The colour-correlated matrix elements have been obtained from the computer code REColA. All singularities (both of QCD and QED origin) from collinear initial-state splittings have been absorbed in the PDFs using the $\overline{\mathrm{MS}}$ factorisation scheme.

Validation: in order to ensure the validity of the calculation, several checks have been performed. The LO hadronic cross section has been compared against the program MADGRAPH5_AMC@NLO [43]. In order to verify the IR and ultra-violet (UV) finiteness, the corresponding regulators have been varied. For each representative partonic channel, the cross sections as well as representative distributions turn out to be independent of such variations. To check the implementation of the subtraction mechanism, the $\alpha$ parame$\operatorname{ter}^{2}[44]$ has been changed from $10^{-2}$ to 1 . This parameter restricts the dipole subtraction

\footnotetext{
${ }^{1}$ Note that RECOLA has been recently implemented in the multi-purpose Monte Carlo codes ShERPA [28] and WHIZARD [29, 30] which allows to compute NLO QCD and EW corrections.

${ }^{2}$ The present computation has been done using the value $\alpha=10^{-2}$.
} 
terms to the vicinity of the singular regions and should drop out of the final results after the inclusion of the corresponding integrated dipoles. This has been checked at both the level of the fiducial cross section and differential distributions. A Ward identity for the gg channel has been verified by calculating for 4000 phase-space points the quantity $\operatorname{Re}\left[\mathcal{M}_{0}^{*}\left(\epsilon_{\mathrm{g}}\right) \mathcal{M}_{1}\left(\epsilon_{\mathrm{g}} \rightarrow p_{\mathrm{g}} / p_{\mathrm{g}}^{0}\right)\right] / \operatorname{Re}\left[\mathcal{M}_{0}^{*}\left(\epsilon_{\mathrm{g}}\right) \mathcal{M}_{1}\left(\epsilon_{\mathrm{g}}\right)\right]$ where one of the initial gluons' polarisation vector $\epsilon_{\mathrm{g}}$ has been replaced by its momentum $p_{\mathrm{g}}$ normalised to its energy $p_{\mathrm{g}}^{0}$. Computing the cumulative fraction of events above certain thresholds gives results comparably good as the ones of refs. $[8,11,12]$. The one-loop matrix elements obtained from RECOLA have been compared against the ones of RECOLA2 [45] where a background-field method [46] formulation of the Standard Model has been implemented. For 4000 phase-space points, it always gave at least 4 digits agreement.

\section{Numerical analysis}

\subsection{Input parameters}

The results presented here are for the LHC running at a centre-of-mass energy of $\sqrt{s}=$ $13 \mathrm{TeV}$. To interface the PDFs, the program LHAPDF $6.1 .5[47,48]$ has been utilised. We have used the NNPDF30_\{lo/nlo\}_as_0118 PDF sets [49] at LO and NLO, respectively. The central value of the factorisation and renormalisation scale has been chosen to be

$$
\mu_{0}=\overline{E_{\mathrm{T}}} / 2=\frac{1}{2} \sqrt{\sqrt{m_{\mathrm{t}}^{2}+p_{\mathrm{T}, \mathrm{t}}^{2}} \sqrt{m_{\mathrm{t}}^{2}+p_{\mathrm{T}, \mathrm{t}}^{2}}}
$$

with $p_{\mathrm{T}, \overline{\mathrm{t}}} / p_{\mathrm{T}, \mathrm{t}}$ standing for the transverse momentum of the top/antitop quark. This choice is motivated by previous computations of off-shell top-antitop production in the fully leptonic channel $[1,3]$. It leads to small scale dependencies and moderate NLO corrections (see figure 4 and related discussion below), as further discussed in sections 4.3 and 4.4 .

The electromagnetic coupling $\alpha$ has been fixed by the Fermi constant in the $G_{\mu}$ scheme $[50]$ as

$$
\alpha=\frac{\sqrt{2}}{\pi} G_{\mu} M_{\mathrm{W}}^{2}\left(1-\frac{M_{\mathrm{W}}^{2}}{M_{\mathrm{Z}}^{2}}\right), \quad \text { with } \quad G_{\mu}=1.16637 \times 10^{-5} \mathrm{GeV}^{2} .
$$

The numerical values of the masses and widths read [51]:

$$
\begin{aligned}
m_{\mathrm{t}} & =173.34 \mathrm{GeV}, & & M_{\mathrm{H}}=125.0 \mathrm{GeV}, \\
M_{\mathrm{Z}}^{\mathrm{OS}} & =91.1876 \mathrm{GeV}, & & \Gamma_{\mathrm{Z}}^{\mathrm{OS}}=2.4952 \mathrm{GeV}, \\
M_{\mathrm{W}}^{\mathrm{OS}} & =80.385 \mathrm{GeV}, & & \Gamma_{\mathrm{W}}^{\mathrm{OS}}=2.085 \mathrm{GeV},
\end{aligned}
$$

with the Higgs-boson mass taken following the recommendations of ref. [52]. The bottom quark is considered massless. The pole masses and widths entering the calculation are determined from the measured on-shell (OS) values [53] for the $\mathrm{W}$ and $\mathrm{Z}$ bosons according to

$$
M_{V}=\frac{M_{V}^{\mathrm{OS}}}{\sqrt{1+\left(\Gamma_{V}^{\mathrm{OS}} / M_{V}^{\mathrm{OS}}\right)^{2}}}, \quad \Gamma_{V}=\frac{\Gamma_{V}^{\mathrm{OS}}}{\sqrt{1+\left(\Gamma_{V}^{\mathrm{OS}} / M_{V}^{\mathrm{OS}}\right)^{2}}} .
$$


The mass and width of the top quark are taken from ref. [54], where $\Gamma_{\mathrm{t}}^{\mathrm{LO}}=1.449582 \mathrm{GeV}$ at $\mathrm{LO}$ and $\Gamma_{\mathrm{t}}^{\mathrm{NLO}}=1.35029 \mathrm{GeV}$ at $\mathrm{NLO} \mathrm{QCD}$, respectively.

\subsection{Event selection}

The event selection is inspired by the searches performed at the LHC by the ATLAS and CMS collaborations in the lepton+jets channel $[19,20]$. The jets (light as well as bottom jets) are clustered with the anti- $k_{\mathrm{T}}$ algorithm [55] using a jet radius of $R=0.4$. A bottom jet clustered with a light jet gives rise to a bottom jet. Note that for photon recombination with charged particles, the clustering radius is taken to be $R=0.1$. The event selection for the final state reads:

$$
\begin{aligned}
\text { light/bottom jets: } & p_{\mathrm{T}, \mathrm{j} / \mathrm{b}}>25 \mathrm{GeV}, & \left|y_{\mathrm{j} / \mathrm{b}}\right| & <2.5, \\
\text { charged lepton: } & p_{\mathrm{T}, \ell}>25 \mathrm{GeV}, & & \left|y_{\ell}\right|<2.5,
\end{aligned}
$$

with $y$ standing for the rapidity. The final state is thus characterised by two light jets, two bottom jets, a charged lepton, and missing energy. This implies that effectively the jet radius is acting as a cut,

$$
\Delta R_{\mathrm{jj}}, \Delta R_{\mathrm{jb}}, \Delta R_{\mathrm{bb}}>0.4
$$

where the distance between two particles $i$ and $j$ is defined as

$$
\Delta R_{i j}=\sqrt{\left(\Delta \phi_{i j}\right)^{2}+\left(\Delta y_{i j}\right)^{2}}
$$

with the azimuthal angle difference $\Delta \phi_{i j}=\min \left(\left|\phi_{i}-\phi_{j}\right|, 2 \pi-\left|\phi_{i}-\phi_{j}\right|\right)$.

This set of cuts aims at measuring top-pair production in the resolved topology as opposed to the boosted topology. ${ }^{3}$ In the resolved event selection, the decay products of the hadronically decaying top quark are required to be separated. The boosted selection, on the other hand, is used for measurements of top quarks with large momenta in association with large- $R$ jets. In order to reduce the non- $t \bar{t}$ background, we have required that at least one jet-jet invariant mass fulfils the criterion

$$
60 \mathrm{GeV}<m_{\mathrm{jj}}<100 \mathrm{GeV}
$$

Hence the two jets are most probably originating from the decay of a $\mathrm{W}$ boson and thus of a top quark. This ensures that the bulk of the cross section is originating mainly from two resonant top quarks and not from background contributions. In particular, it removes real radiation events where the two jets originating from the W-boson decay are recombined into a single jet, while the extra real radiation gives rise to the presence of a second separated jet. Such events typically have boosted kinematics and can make the real contribution potentially very large. This effect of quarks being recombined at high transverse momentum has already been foreseen in ref. [56].

\footnotetext{
${ }^{3}$ Such a distinction is for example made by the ATLAS collaboration in ref. [19].
} 


\begin{tabular}{|cccc|}
\hline Ch. & $\sigma_{\mathrm{LO}}[\mathrm{pb}]$ & $\sigma_{\mathrm{NLO}}[\mathrm{pb}]$ & $K$-factor \\
\hline $\mathrm{gg}$ & $12.0257(5)$ & $13.02(7)$ & 1.08 \\
$q \bar{q}$ & $1.3308(3)$ & $0.942(7)$ & 0.71 \\
$\mathrm{~g} q(/ \bar{q})$ & & $1.604(5)$ & \\
\hline $\mathrm{pp}$ & $13.3565(6)$ & $15.56(7)$ & 1.16 \\
\hline
\end{tabular}

Table 1. Fiducial cross sections at LO and NLO for the process pp $\rightarrow \mu^{-} \bar{\nu}_{\mu}$ bbjj with its corresponding sub-channels. The possible flavours of the quark are $q=\mathrm{u}, \mathrm{d}, \mathrm{c}, \mathrm{s}$. The quark-gluon channels denoted by $\mathrm{g} q(/ \bar{q})$ consist in the real corrections with gluon-quark initial states in eq. (3.1) and appear only at NLO. The proton-proton cross section is presented in the last line of the table dubbed pp. The $K$-factors are defined as $K=\sigma_{\mathrm{NLO}} / \sigma_{\mathrm{LO}}$. The integration errors of the last digits are given in parentheses. The predictions are expressed in pb and are for the LHC running at a centre-of-mass energy of $\sqrt{s}=13 \mathrm{TeV}$.

\subsection{Cross sections}

In table 1, the fiducial cross sections at both LO and NLO are presented. At LO, the gg channel amounts to $90.0 \%$ of the fiducial cross section while the $q \bar{q}$ ones account for $10.0 \%$. The contributions with bottom quarks in the initial state turn out to be completely negligible and are around a per mille ( $0.13 \%$ of the LO cross section) which is the Monte Carlo error of our NLO calculation. Therefore, these contributions have been omitted in the predictions presented here. At NLO, the gg channel represents $83.7 \%$ of the fiducial cross section while the $q \bar{q}$ one is amounting to $6.0 \%$. At NLO, the real corrections with gluon-quark initial states in eq. (3.1) are accounting for $10.3 \%$ of the cross section.

It is worth noting that the $K$-factor of the gluonic channel is larger than the one for quark-antiquark annihilation, amounting to 1.08 and 0.71 , respectively. Different $K$-factors for different partonic channels have already been observed for similar processes $[1,3,11,57$ 59]. Owing to the addition of the $g q(/ \bar{q})$ channels of eq. (3.1) at NLO, the $K$-factor of the fiducial cross section is 1.16 .

The effect of the variation of the factorisation and renormalisation scales on the total prediction has been studied. To this end, the central value $\mu=\overline{E_{\mathrm{T}}} / 2$ has been re-scaled by factors $\xi_{\text {fac }}$ and $\xi_{\text {ren }}$ for

$$
\left(\xi_{\text {fac }}, \xi_{\text {ren }}\right) \in\{(1 / 2,1 / 2),(1 / 2,1),(1,1 / 2),(1,1),(1,2),(2,1),(2,2)\},
$$

where $\left(\xi_{\text {fac }}, \xi_{\text {ren }}\right)=(1,1)$ represents the central scale. In addition to the cross sections for the central scale reported in table 1 , the lowest and highest cross sections for the combinations of eq. (4.9) have been extracted. The fiducial cross sections with scale variation read

$$
\sigma_{\mathrm{LO}}=13.3565(6)_{-22.09 \%}^{+30.68 \%} \mathrm{pb}
$$

and

$$
\sigma_{\mathrm{NLO}}=15.56(7)_{-4.6(5) \%}^{+0.9(6) \%} \mathrm{pb},
$$

where the values in per cent represent the extrema of the cross sections calculated for the scales (4.9). As expected, there is a significant reduction of the scale uncertainty (more than 


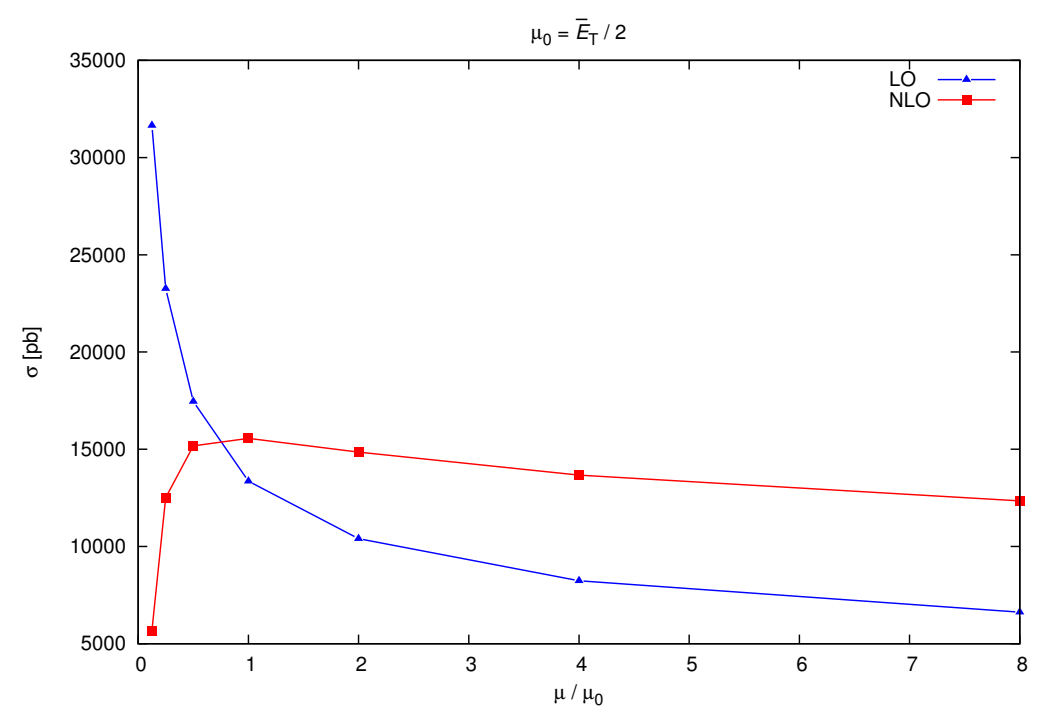

Figure 4. Scale dependence of the LO and NLO cross sections at a centre-of-mass energy $\sqrt{s}=$ $13 \mathrm{TeV}$ at the LHC for $\mathrm{pp} \rightarrow \mu^{-} \bar{\nu}_{\mu} \mathrm{b} \overline{\mathrm{b} j \mathrm{j}}$. The renormalisation and factorisation scales are varied together around the central scale $\mu_{0}=\overline{E_{\mathrm{T}}} / 2$ defined in eq. (4.1).

a factor four) when going from LO to NLO accuracy. The asymmetric scale uncertainty at NLO is due to our choice of the central scale near the maximum of the cross section. The positive uncertainty in eq. (4.11) results in fact from off-diagonal scale variations.

In figure 4 the fiducial cross section at both LO and NLO accuracy is given as a function of the ratio of scales $\mu / \mu_{0}$ in the range $[1 / 8,8]$. Both the factorisation and renormalisation scales are set equal to the scale $\mu$, while the scale $\mu_{0}=\overline{E_{\mathrm{T}}} / 2$ is defined in eq. (4.1). The LO cross section shows the usual unbounded exponential behaviour, while the NLO prediction displays a much smaller scale dependence. Near $\mu_{0}=\overline{E_{\mathrm{T}}} / 2$ the NLO cross section is flat, and the scale variation is minimal. Hence, the choice $\mu=\mu_{0}$ ensures a maximal reduction of the scale uncertainty when going from LO to NLO for the fiducial cross section. Note however, that the resulting small positive scale variation tends to underestimate the uncertainty in this case and the choice $\mu_{0}=\overline{E_{\mathrm{T}}}$ would provide a more conservative NLO scale uncertainty. While for $\mu_{0}=\overline{E_{\mathrm{T}}} / 2$ the difference between LO and NLO predictions is reasonably small, guaranteeing that the NLO cross section is within LO scale uncertainty, this is not the case for the choice $\mu_{0}=\overline{E_{\mathrm{T}}}$ which leads to much larger NLO corrections. In any case, the NLO cross section has a good perturbative behaviour and provides a much more reliable prediction.

Finally, we recall that this computation involves only the partonic channels that feature two resonant top quarks and thus two resonant W bosons [see eq. (2.2)]. All other partonic channels receive only contributions with one resonant top quark and one resonant $\mathrm{W}$ boson. Imposing a cut on the invariant mass of the two jets around the W-boson mass additionally suppresses these contributions both at the level of the cross section and differential distributions. ${ }^{4}$ It thus renders these partonic channels phenomenologically negligible.

\footnotetext{
${ }^{4}$ In the next section, comments are made for differential distributions where the effect of non-doublytop-resonant partonic channels is larger than $1 \%$.
} 


\subsection{Differential distributions}

Turning to the differential distributions, for each of them, the LO and NLO predictions are shown in the upper plot while the ratio of the two predictions is presented in the lower panel. The band is obtained by variation of the factorisation and renormalisation scales independently within the set of eq. (4.9). In the NLO/LO ratio, the predictions are normalised to the LO ones for the central scale.

Transverse-momentum distributions. In figure 5, several transverse-momentum distributions are shown. The first two, in figures $5 \mathrm{a}$ and $5 \mathrm{~b}$, are the ones for the hardest and second hardest jet, ${ }^{5}$ respectively. In figure 5 a, one observes a strong increase of the NLO corrections towards high transverse momentum. Below $150 \mathrm{GeV}$, the corrections stay below $100 \%$ while above they become more than one order of magnitude larger. At $400 \mathrm{GeV}$, the NLO prediction is 31 times larger than the LO prediction. This is a purely kinematical effect in combination with the event selection which explains why the scale-variation band is not a reliable estimate here. Since the LO contribution is strongly suppressed for high transverse jet momenta, the NLO scale-variation band increases towards high transverse momentum. There, the two jets originating from the $\mathrm{W}$ boson are collinear. Consequently at LO such configurations are forbidden due to the jet distance cut (4.6), while at NLO such events are allowed owing to the extra jet from real emission. Hence these large corrections are due to a suppression of the LO configuration in this phase-space region. This effect is particularly explicit for the transverse momentum of the second hardest jet. At $200 \mathrm{GeV}$, the LO predictions decrease sharply to become even zero around $250 \mathrm{GeV}$ because of the invariant-mass cut and the $\Delta R$ cut. Indeed, assuming small angles between the two jets leads because of $p_{\mathrm{T}, \mathrm{j}_{2}}<p_{\mathrm{T}, \mathrm{j}_{1}}$ to $p_{\mathrm{T}, \mathrm{j}_{2}, \text { max }}^{2} \sim m_{\mathrm{jj}, \text { max }}^{2} / \Delta R_{\mathrm{jj} \text {, min }}^{2}=(100)^{2} /(0.4)^{2}=(250 \mathrm{GeV})^{2}$. A similar behaviour has already been observed in ref. [60] when considering doubly top-resonant contributions. Above this threshold, the NLO contributions consist exclusively of real radiation where the two jets originating from the $\mathrm{W}$-boson decay are recombined in a single jet. Such events are accepted if the invariant mass of this jet and the real radiation jet is still fulfilling the requirement of eq. (4.8). Without this requirement, the contribution of such real radiation events would be much larger.

The transverse momenta of the leptonic and hadronic top quarks are displayed in figures $5 \mathrm{c}$ and $5 \mathrm{~d}$, respectively. The definition of the leptonic top quark is based on Monte Carlo truth and is thus the total momentum of the anti-bottom quark (possibly recombined with a light jet), the charged lepton, and the neutrino. At low transverse momentum, the corrections are at the level of $26 \%$. They are below $10 \%$ at $150 \mathrm{GeV}$ and increase steadily to reach $35 \%$ at $400 \mathrm{GeV}$. On the other hand, the definition of the reconstructed hadronic top quark reads: from the two or three light jets, retain the two momenta whose combination has the invariant mass closest to the W-boson mass. Out of the two bottom quarks, retain the one whose momentum when added to the ones of the two pre-selected jets results in a 3 -jet invariant mass closest to the top-quark mass. The total momentum of these two light jets and bottom jet defines the reconstructed hadronic top

\footnotetext{
${ }^{5}$ The hardest jet is the one with the highest transverse momentum, etc.
} 
(a)
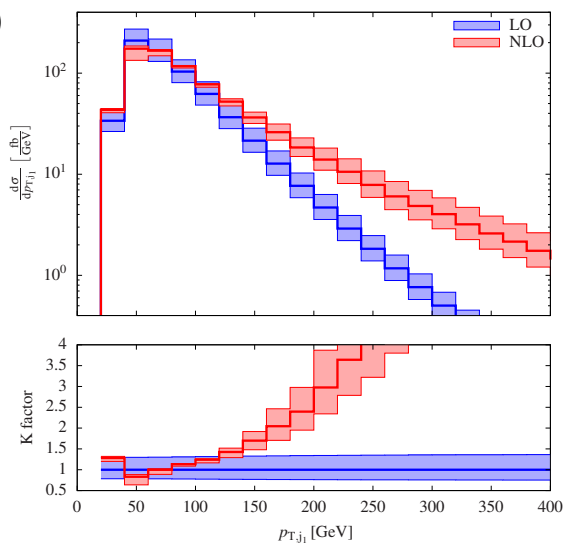

(c)
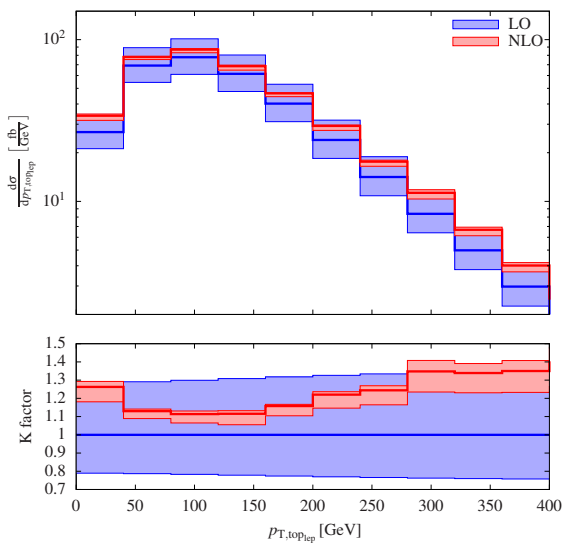

(e)
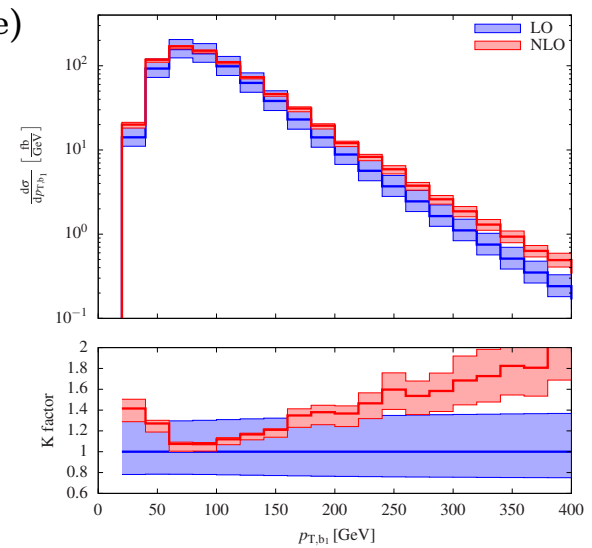

(b)
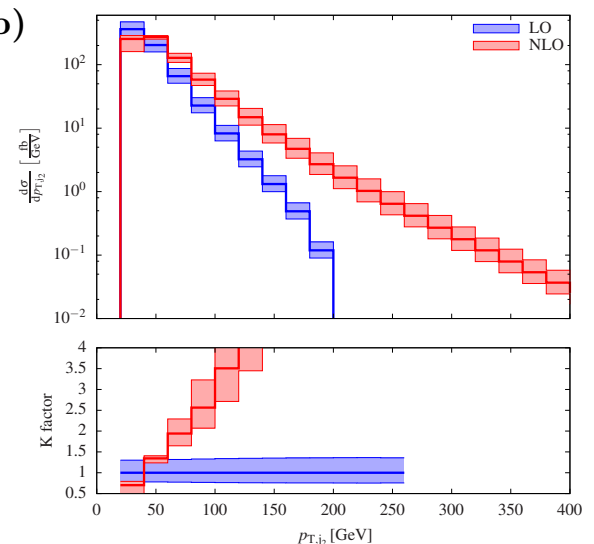

(d)
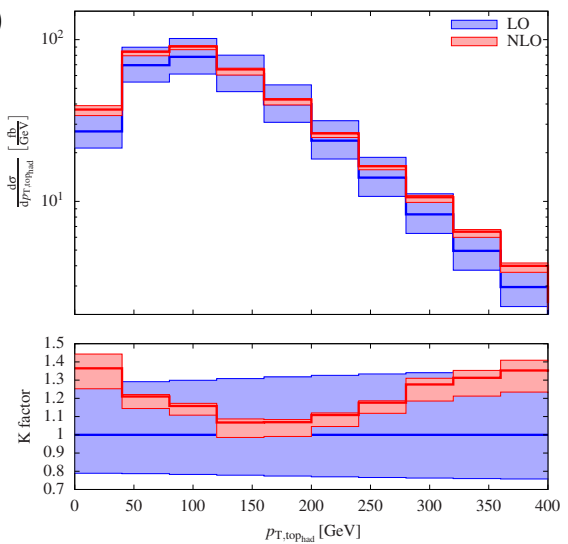

(f)
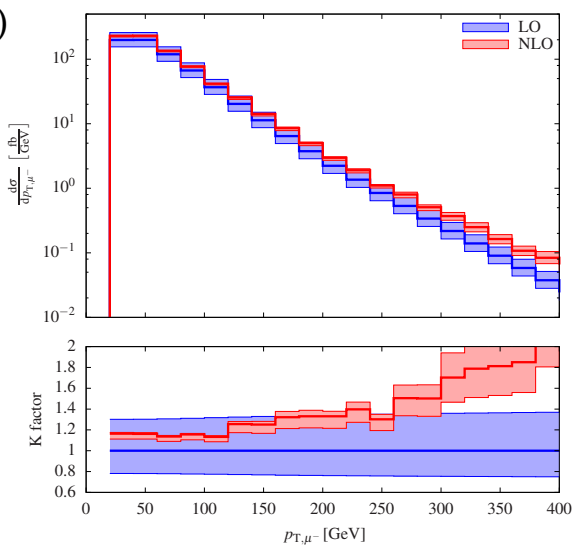

Figure 5. Differential distributions at a centre-of-mass energy $\sqrt{s}=13 \mathrm{TeV}$ at the LHC for $\mathrm{pp} \rightarrow \mu^{-} \bar{\nu}_{\mu} \mathrm{b} \overline{\mathrm{b} j \mathrm{j}}$ : (a) transverse momentum of hardest jet (top left), (b) transverse momentum of the second hardest jet (top right), (c) transverse momentum of the leptonic top quark (middle left), (d) transverse momentum of the reconstructed hadronic top quark (middle right), (e) transverse momentum of the hardest bottom jet (bottom left), and (f) transverse momentum of the muon (bottom right). The upper panels show the LO prediction as well as the NLO one. The lower panels display the ratio of the NLO and the LO predictions. The bands correspond to factor- 2 scale variations as defined in eq. (4.9). 
momentum. The behaviour of the corrections is comparable to the one for the leptonic top quark. The corrections are around $37 \%$ at zero transverse momentum, reach a minimum at $150 \mathrm{GeV}$, and are maximal at high transverse momentum where the effect described above is taking place. At $400 \mathrm{GeV}$, the NLO corrections amount to $35 \%$. This behaviour has already been observed for top-pair production with purely leptonic decays [3]. The increased corrections for small transverse momenta result from a redistribution of events where a gluon is emitted from one of the decay products of the top quark and carries away momentum. This effect is amplified in the case of hadronic top quark owing to the three jets in the final state that can radiate gluons while for the leptonic top quark, only one jet is present in the decay products. The transverse-momentum distributions for the top quarks are more inclusive than the ones for the jets in the sense that they are built from more momenta. Therefore, they are less sensitive to the kinematical effect described previously. This explains why they are slowly decreasing towards higher transverse momentum and do not display extremely large corrections over the phase space studied. Nonetheless the $K$-factor is not flat and becomes sizeable both for low and high transverse momenta.

The distribution in the transverse momentum of the hardest bottom jet, figure 5e, exhibits a similar behaviour as the one in the transverse momentum of the leading jet, figure 5a, but much less pronounced. Again, the corrections increase in the tail of the distribution where non-resonant contributions become relevant. There, the production of the $\mu^{-} \bar{\nu}_{\mu}$ b $\overline{b j j}$ final state proceeds increasingly through contributions that do not feature two resonant top quarks $[8,60]$. The increase of the corrections for low transverse momentum is correlated with the decrease of the LO predictions. Finally, figure $5 \mathrm{f}$ displays the transverse momentum of the muon. Apart from the small transverse-momentum region, this observable shows a similar behaviour as the transverse momentum of the hardest bottom jet. The corrections start below $20 \%$ near the cut at $30 \mathrm{GeV}$ to increase smoothly up to a $K$-factor of 2.7 at $400 \mathrm{GeV}$. This increase of the NLO corrections towards high transverse momentum is also accompanied by an increase of the size of the scale-variation band.

The contributions from non-doubly-top-resonant partonic channels are typically increasing towards high transverse momenta. For the distributions in the transverse momentum of the hardest jet and the hardest bottom jet, they exceed $1 \%$ at $300 \mathrm{GeV}$ and amount to $5 \%$ at $500 \mathrm{GeV}$. For the distribution in the transverse momentum of the second hardest jet, they quickly increase up to $4 \%$ around $200 \mathrm{GeV}$, i.e. above the kinematical threshold described above. On the other hand, for the distributions in the transverse momentum of the top quarks (either leptonic or hadronic), these effects are below $1 \%$ up to at least $500 \mathrm{GeV}$ as these observables are more inclusive than those in the transverse momentum of single particles.

Invariant-mass distributions. In figure 6 four invariant-mass distributions are displayed. Figure 6a shows the invariant mass of leptonically decaying top quark based on Monte Carlo truth. The well-known radiative tail below the top-quark resonance (see, for instance, ref. [3] for the off-shell production of top-quark pairs that decay fully leptonically) is due to final-state radiation that is not reconstructed with the decay products of the top quark and thus not taken into account in the definition of the top-quark invariant mass. 
(a)
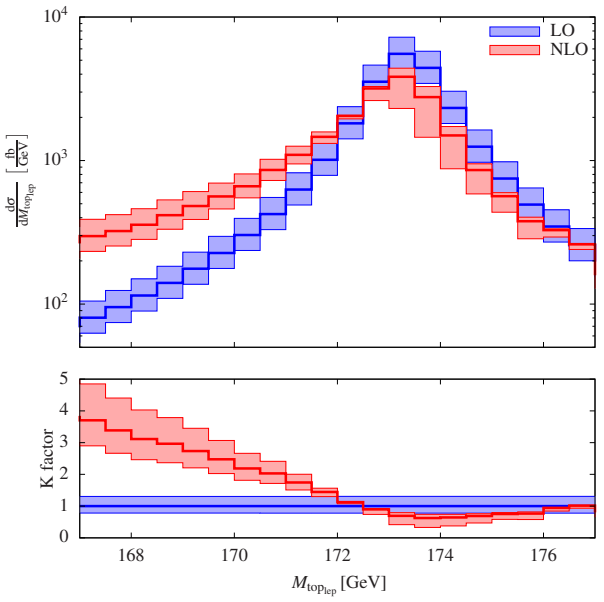

(c)
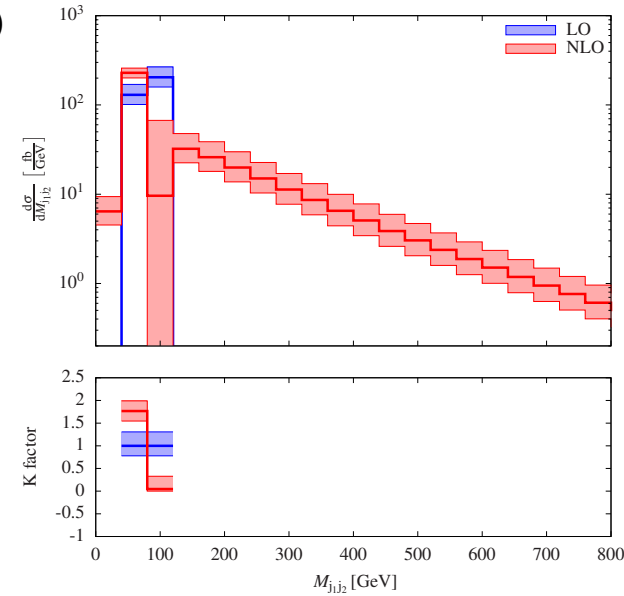

(b)
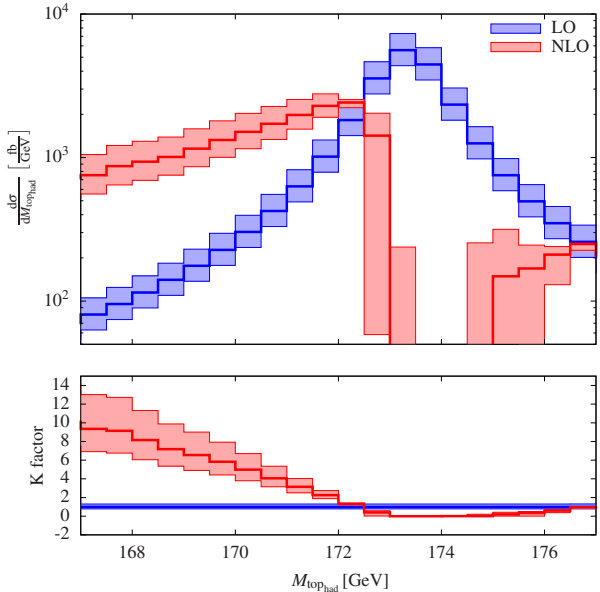

(d)
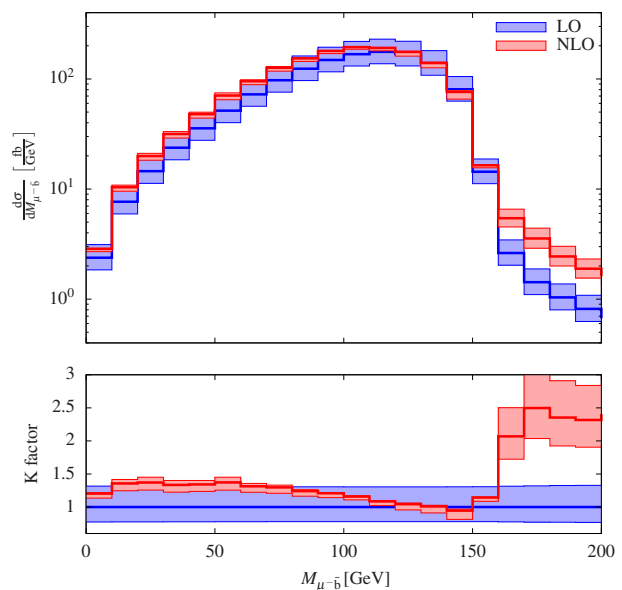

Figure 6. Differential distributions at a centre-of-mass energy $\sqrt{s}=13 \mathrm{TeV}$ at the LHC for pp $\rightarrow \mu^{-} \bar{\nu}_{\mu}$ bbjjj: (a) invariant mass of the leptonic top quark (top left), (b) invariant mass of the hadronic top quark (top right), (c) invariant mass of the two hardest jets (bottom left), and (d) invariant mass of the muon and anti-bottom quark (bottom right). The upper panels show the LO prediction as well as the NLO one. The lower panels display the ratio of the NLO and the LO predictions. The bands correspond to factor-2 scale variations as defined in eq. (4.9).

Hence, events that are close to resonance at LO tend to be shifted below the resonance peak when the extra real radiation is not soft or collinear to the bottom quark. Note that on the peak the corrections can reach about $-35 \%$. For the invariant mass of the hadronic top, this effect is enhanced owing to three QCD jets in the final state (two light jets and one bottom jet) that can radiate gluons instead of one bottom jet for the leptonic case. As shown in figure $6 \mathrm{~b}$, this results in negative NLO corrections of more than $100 \%$ in some bins close to the resonance. A proper description of this distribution thus requires the inclusion of higher-order corrections, which is beyond the scope of this work.

In figure $6 \mathrm{c}$, the distribution in the invariant mass of the two hardest jets (not necessarily the two jets that enter the definition of the hadronic top quark) is displayed. At LO, 
the di-jet invariant-mass is restricted to the range $60-100 \mathrm{GeV}$ owing to the invariant-mass cut on the di-jet system [see eq. (4.8)]. As explained above, this condition is relaxed at NLO due to the appearance of a third jet in the real emission. Hence, these real contributions contribute in a wider part of phase space, while the LO and virtual contributions are restricted to the mass window $60-100 \mathrm{GeV}$. As a consequence the cancellation of enhanced IR-sensitive terms between virtual and real corrections is not happening locally. In particular, this mechanism can lead to locally negative predictions at the kinematical boundaries [61] which is particularly apparent here in the bin between $80 \mathrm{GeV}$ and $120 \mathrm{GeV}$.

The distribution in the invariant mass of the muon and the anti-bottom quark is presented in figure $6 \mathrm{~d}$. This observable has been intensively investigated both in experiments [62] and in theory $[16,17]$ as it has been identified [3] to be very sensitive to the value of the top-quark mass. Indeed, the sharp decrease at $154 \mathrm{GeV}$ represents the transition from on-shell production of top quarks to a region dominated by non-doubly-top-resonant contributions. ${ }^{6}$ For on-shell top quark and $\mathrm{W}$ boson, this invariant mass is bounded at $M_{\mu^{-} \overline{\mathrm{b}}}^{2}=M_{\mathrm{t}}^{2}-M_{\mathrm{W}}^{2} \simeq(154 \mathrm{GeV})^{2}$. Below this limit, the NLO corrections vary from $+35 \%$ at $50 \mathrm{GeV}$ to $-5 \%$ at $150 \mathrm{GeV}$ while the $K$-factor increases up to 2.5 above this limit. This is simply due to the fact that this region is dominated by non-resonant contributions which receive large corrections as explained previously. Hence the NLO corrections computed here are important and should have an impact on the top-quark mass determination based on such an observable [16, 17].

Angular and rapidity distributions. Finally, some angular and rapidity distributions are presented in figure 7. In figure $7 \mathrm{a}$ we show the distribution in the rapidity-azimuthalangle distance between the two hardest jets defined as in eq. (4.7). At LO, the distribution is abruptly decreasing above $\pi$ and even going to zero above $4 .{ }^{7}$ Using again the approximation for small angles gives $\Delta R_{\mathrm{jj}, \text { max }}^{2} \sim m_{\mathrm{jj}, \max }^{2} / p_{\mathrm{T}, \mathrm{j}, \min }^{2}=(100)^{2} /(25)^{2}=4^{2}$. Above this point, the LO contributions are forbidden due to the event selection, but this is relaxed at NLO owing to the appearance of real radiation as for the previously discussed distributions. As a consequence the NLO contributions are dominating in this region.

Next, we present distributions in the azimuthal angle and in the cosine of the angle between the hardest jet and the muon in figures $7 \mathrm{~b}$ and $7 \mathrm{c}$, respectively. Both distributions are smooth and relatively flat. Therefore the NLO corrections are pretty stable over the whole range. They essentially feature the normalisation present at the level of the fiducial cross section. In these distributions the reduction of the scale uncertainty is particularly visible demonstrating the need for NLO predictions.

We finish with rapidity distributions. Figure $7 \mathrm{~d}$ shows the rapidity distribution of the reconstructed hadronic top quark, defined as for the transverse-momentum distribution shown in figure $5 \mathrm{~d}$. The corrections are smaller in the central region where the two top quarks are mainly produced on shell. In the peripheral region, the non-resonant contributions come into play and thus lead to larger corrections. Finally, we present the rapidity

\footnotetext{
${ }^{6}$ Above the threshold, the effect of non-doubly-top-resonant partonic channels amounts to $2-3 \%$.

${ }^{7}$ Above $\Delta R_{\mathrm{j}_{1} \mathrm{j}_{2}}=3.5$, the contribution of non-doubly-top-resonant partonic channels rises strongly to more than $5 \%$.
} 
(a)

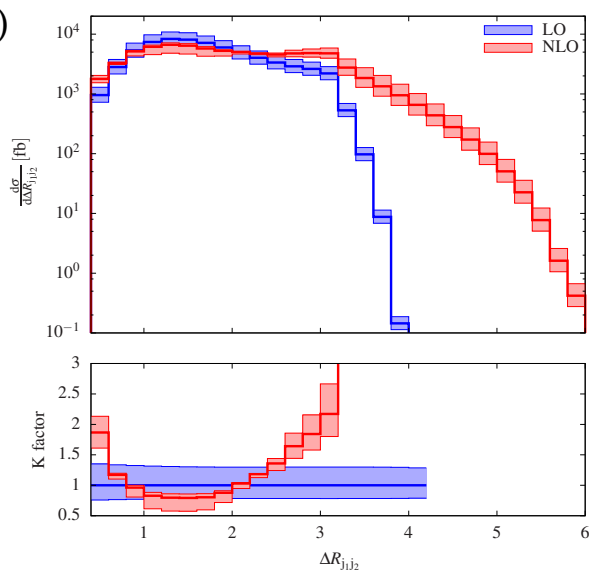

(c)
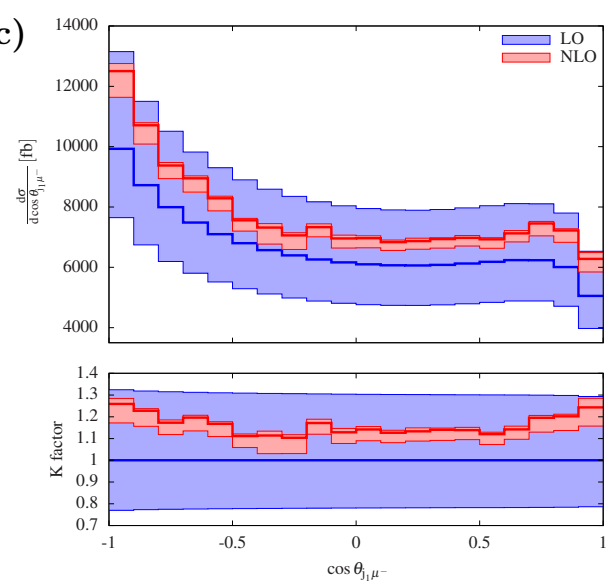

(e)
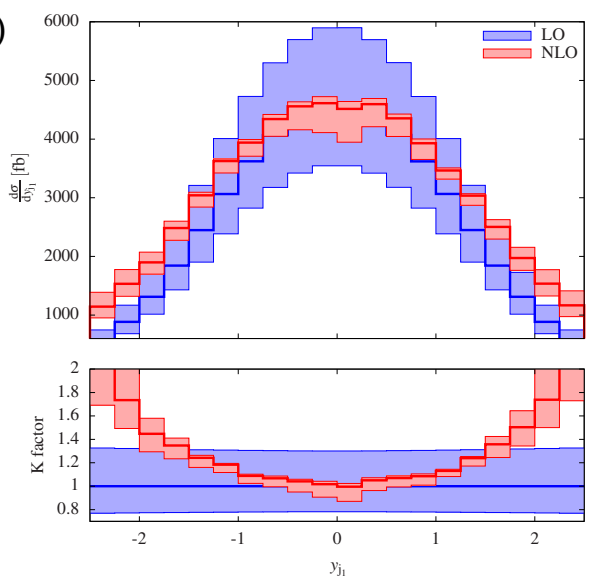

(b)

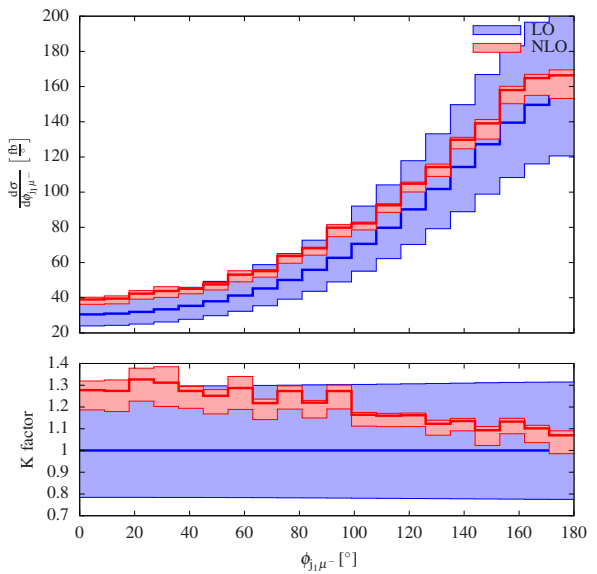

(d)
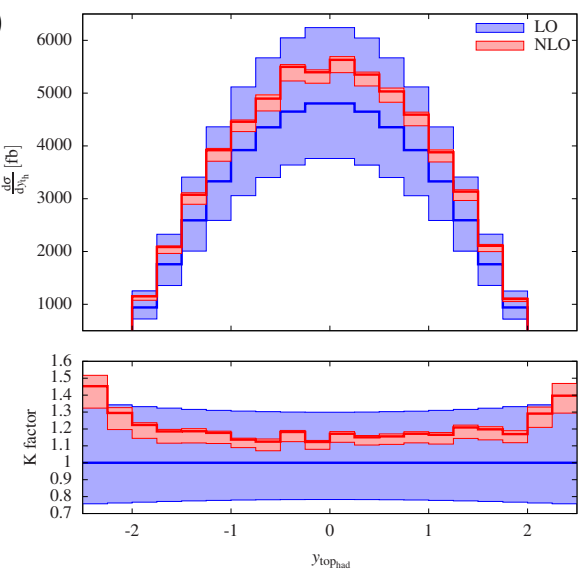

(f)
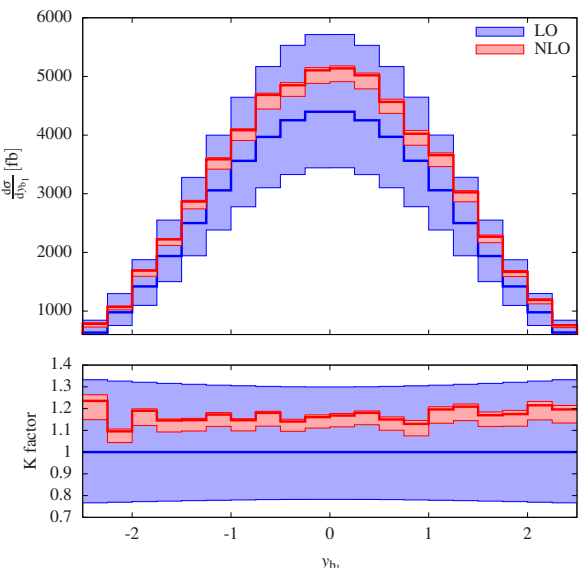

Figure 7. Differential distributions at a centre-of-mass energy $\sqrt{s}=13 \mathrm{TeV}$ at the LHC for pp $\rightarrow \mu^{-} \bar{\nu}_{\mu}$ b $\overline{b j j: ~(a) ~ r a p i d i t y-a z i m u t h a l-a n g l e ~ d i s t a n c e ~ b e t w e e n ~ t h e ~ t w o ~ h a r d e s t ~ j e t s ~(t o p ~ l e f t), ~}$ (b) azimuthal angle between the hardest jet and the muon (top right), (c) cosine of the angle between the hardest jet and the muon (middle left). (d) rapidity of the reconstructed hadronic top quark (middle right), (e) rapidity of the hardest light jet (bottom left), and (f) rapidity of the hardest bottom jet (bottom right). The upper panels show the LO prediction as well as the NLO one. The lower panels display the ratio of the NLO and the LO predictions. The bands correspond to factor-2 scale variations as defined in eq. (4.9). 
distributions of the hardest light and hardest bottom jet in figures $7 \mathrm{e}$ and $7 \mathrm{f}$, respectively. The NLO corrections to the rapidity of the hardest jet vary stronger than those for the hadronic top quark. They reach $100 \%$ at a rapidity of \pm 2.5 while they are close to zero in the central region. In particular for $\left|y_{\mathrm{j}_{1}}\right|>2$ the LO and NLO uncertainty bands do not overlap, and the NLO uncertainty band becomes larger in this phase-space region. Large corrections, which can be attributed to real emission of jets from the incoming partons, show up in this phase-space region such that the accuracy of our prediction is effectively only of LO. On the other hand, the distribution in the rapidity of the hardest bottom jet does not display significant shape distortions owing NLO corrections over the whole kinematical range and merely inherits the correction factor for the fiducial cross section.

\section{Conclusion}

The production of two top quarks gives rise to three different classes of final states, depending on whether the two $\mathrm{W}$ bosons and thus the two top-quarks decay leptonically or hadronically. So far, most of the theoretical work has focused on the channel where the two top quarks decay leptonically. Nonetheless, the lepton+jets channel where one top quark decays hadronically and the other leptonically possesses some advantages over the fully leptonic channel. First, it has a larger cross section due to the hadronic branching ratio of the $\mathrm{W}$ boson and second, it allows for a better detection and reconstruction of top quarks. Indeed, in this case only one neutrino in the final state leads to missing transverse energy. Hence, the process pp $\rightarrow \mu^{-} \bar{\nu}_{\mu}$ bbjj constitutes one of the key channels for the study of the top-quark properties at the LHC, and thus precise predictions for it are highly desirable.

So far, the full process was only known at LO. At NLO, the best available predictions included NLO corrections to the on-shell production and LO decay of the top quarks. For the first time we have computed the NLO QCD corrections to the off-shell process pp $\rightarrow$ $\mu^{-} \bar{\nu}_{\mu} \mathrm{b} \overline{b j j}$ for all partonic channels that feature doubly-resonant top quarks. The calculation features by definition off-shell and non-resonant effects. These effects are becoming more and more relevant for run II of the LHC, where a large amount of data is collected at the increased energy of $13 \mathrm{TeV}$. Hence the high-energy region where these effects are more important will be accurately probed in the future, making such computations very relevant. In addition, in the present computation the event selection applied to the final state mimics the one used by the experimental collaborations in order to provide realistic predictions. We have focussed on the phase space relevant for top-pair production and omitted partonic channels that involve only one resonant top quark and one resonant $\mathrm{W}$ boson as well as bottom-quark-initiated and photon-induced contributions. The corresponding leadingorder contributions have been shown to be negligible in the phase space relevant for top-pair production.

The results are different from those for leptonically decaying top quarks, and the NLO corrections are particularly large in certain phase-space regions. These large corrections arise in high-energy regions where contributions without two resonant top quarks are important. This is particularly explicit in the tails of the transverse-momentum distributions. Also, for observables that feature a kinematic threshold, above this threshold the NLO cor- 
rections are particularly large. This happens for example for the distance between the two hardest jets and for the invariant mass of the muon and anti-bottom which is important for the top-quark mass determination.

The NLO corrections are very sensitive to the experimental event selection. In particular, the jet radius is a key parameter as it ensures that the jets are separated in the resolved-topology event selection. Increasing or decreasing the jet radius would affect the NLO corrections accordingly. In addition, we have applied a cut on the di-jet invariant mass ensuring that a jet pair originates most probably from a $\mathrm{W}$ boson and thus indirectly from a top quark. This cut, in particular, removes events at high transverse momentum where the two jets originating from the $\mathrm{W}$ boson are recombined in one jet while the extra real radiation ensures the presence of two separated jet. In this way, mainly doubly-resonant top-quark contributions in the resolved topology are selected at both LO and NLO.

On the technical side, the present NLO computation is non-trivial as it possesses four coloured particles in addition to two leptons in the final state. Such a computation has been made possible by the use of the matrix-element generator RECOLA in combination with the Collier library as well as an efficient Monte Carlo program dubbed MoCaNLO.

Finally, as the computation uses experimental event selection for the final states, this should allow the experimental collaborations to include these corrections in their forthcoming analysis.

\section{Acknowledgments}

We are thankful to Jean-Nicolas Lang and Sandro Uccirati for supporting RECOLA and Robert Feger concerning MoCANLO. We thank Mauro Chiesa for performing some checks of the calculation and Manfred Kraus for useful discussions. We acknowledge financial support by the German Federal Ministry for Education and Research (BMBF) under contract no. 05H15WWCA1 and the German Science Foundation (DFG) under reference number DE 623/6-1.

Open Access. This article is distributed under the terms of the Creative Commons Attribution License (CC-BY 4.0), which permits any use, distribution and reproduction in any medium, provided the original author(s) and source are credited.

\section{References}

[1] A. Denner, S. Dittmaier, S. Kallweit and S. Pozzorini, NLO QCD corrections to $W W b b$ production at hadron colliders, Phys. Rev. Lett. 106 (2011) 052001 [arXiv:1012.3975] [INSPIRE].

[2] G. Bevilacqua, M. Czakon, A. van Hameren, C.G. Papadopoulos and M. Worek, Complete off-shell effects in top quark pair hadroproduction with leptonic decay at next-to-leading order, JHEP 02 (2011) 083 [arXiv: 1012.4230] [INSPIRE].

[3] A. Denner, S. Dittmaier, S. Kallweit and S. Pozzorini, NLO QCD corrections to off-shell top-antitop production with leptonic decays at hadron colliders, JHEP 10 (2012) 110 [arXiv: 1207.5018] [INSPIRE]. 
[4] R. Frederix, Top Quark Induced Backgrounds to Higgs Production in the $W W^{(*)} \rightarrow l l \nu \nu$ Decay Channel at Next-to-Leading-Order in QCD, Phys. Rev. Lett. 112 (2014) 082002 [arXiv: 1311.4893] [INSPIRE].

[5] F. Cascioli, S. Kallweit, P. Maierhöfer and S. Pozzorini, A unified NLO description of top-pair and associated Wt production, Eur. Phys. J. C 74 (2014) 2783 [arXiv:1312.0546] [INSPIRE].

[6] J.M. Campbell, R.K. Ellis, P. Nason and E. Re, Top-pair production and decay at NLO matched with parton showers, JHEP 04 (2015) 114 [arXiv: 1412.1828] [INSPIRE].

[7] T. Ježo, J.M. Lindert, P. Nason, C. Oleari and S. Pozzorini, An NLO+PS generator for $t \bar{t}$ and $W t$ production and decay including non-resonant and interference effects, Eur. Phys. J. C 76 (2016) 691 [arXiv: 1607.04538] [INSPIRE].

[8] A. Denner and M. Pellen, NLO electroweak corrections to off-shell top-antitop production with leptonic decays at the LHC, JHEP 08 (2016) 155 [arXiv:1607.05571] [INSPIRE].

[9] G. Bevilacqua, H.B. Hartanto, M. Kraus and M. Worek, Top Quark Pair Production in Association with a Jet with Next-to-Leading-Order QCD Off-Shell Effects at the Large Hadron Collider, Phys. Rev. Lett. 116 (2016) 052003 [arXiv: 1509.09242] [INSPIRE].

[10] G. Bevilacqua, H.B. Hartanto, M. Kraus and M. Worek, Off-shell top quarks with one jet at the LHC: a comprehensive analysis at NLO QCD, JHEP 11 (2016) 098 [arXiv:1609.01659] [INSPIRE].

[11] A. Denner and R. Feger, NLO QCD corrections to off-shell top-antitop production with leptonic decays in association with a Higgs boson at the LHC, JHEP 11 (2015) 209 [arXiv: 1506.07448] [INSPIRE].

[12] A. Denner, J.-N. Lang, M. Pellen and S. Uccirati, Higgs production in association with off-shell top-antitop pairs at NLO EW and QCD at the LHC, JHEP 02 (2017) 053 [arXiv: 1612.07138] [INSPIRE].

[13] J. Gao and A.S. Papanastasiou, Top-quark pair-production and decay at high precision, Phys. Rev. D 96 (2017) 051501 [arXiv:1705.08903] [INSPIRE].

[14] M. Czakon, P. Fiedler, D. Heymes and A. Mitov, NNLO QCD predictions for fully-differential top-quark pair production at the Tevatron, JHEP 05 (2016) 034 [arXiv: 1601.05375] [INSPIRE].

[15] M. Czakon, D. Heymes and A. Mitov, Dynamical scales for multi-TeV top-pair production at the LHC, JHEP 04 (2017) 071 [arXiv: 1606.03350] [INSPIRE].

[16] G. Heinrich, A. Maier, R. Nisius, J. Schlenk and J. Winter, NLO QCD corrections to $W^{+} W^{-} b \bar{b}$ production with leptonic decays in the light of top quark mass and asymmetry measurements, JHEP 06 (2014) 158 [arXiv: 1312.6659] [INSPIRE].

[17] G. Heinrich, A. Maier, R. Nisius, J. Schlenk, M. Schulze, L. Scyboz et al., NLO and off-shell effects in top quark mass determinations, arXiv:1709.08615 [INSPIRE].

[18] G. Bevilacqua, H.B. Hartanto, M. Kraus, M. Schulze and M. Worek, Top quark mass studies with $t \bar{t} j$ at the $L H C$, arXiv:1710.07515 [INSPIRE].

[19] ATLAS collaboration, Measurements of top-quark pair differential cross-sections in the lepton+jets channel in pp collisions at $\sqrt{s}=13$ TeV using the ATLAS detector, JHEP 11 (2017) 191 [arXiv: 1708.00727] [INSPIRE]. 
[20] CMS collaboration, Measurement of differential cross sections for top quark pair production using the lepton+jets final state in proton-proton collisions at 13 TeV, Phys. Rev. D 95 (2017) 092001 [arXiv:1610.04191] [INSPIRE].

[21] A. Manohar, P. Nason, G.P. Salam and G. Zanderighi, How bright is the proton? A precise determination of the photon parton distribution function, Phys. Rev. Lett. 117 (2016) 242002 [arXiv: 1607.04266] [INSPIRE].

[22] B. Biedermann, A. Denner and M. Pellen, Complete NLO corrections to $W^{+} W^{+}$scattering and its irreducible background at the LHC, JHEP 10 (2017) 124 [arXiv:1708.00268] [INSPIRE].

[23] F.A. Berends, R. Pittau and R. Kleiss, All electroweak four fermion processes in electron positron collisions, Nucl. Phys. B 424 (1994) 308 [hep-ph/9404313] [INSPIRE].

[24] A. Denner, S. Dittmaier, M. Roth and D. Wackeroth, Predictions for all processes $e^{+} e^{-} \rightarrow 4$ fermions $+\gamma$, Nucl. Phys. B 560 (1999) 33 [hep-ph/9904472] [INSPIRE].

[25] S. Dittmaier and M. Roth, LUSIFER: A LUcid approach to six FERmion production, Nucl. Phys. B 642 (2002) 307 [hep-ph/0206070] [INSPIRE].

[26] S. Actis, A. Denner, L. Hofer, A. Scharf and S. Uccirati, Recursive generation of one-loop amplitudes in the Standard Model, JHEP 04 (2013) 037 [arXiv:1211.6316] [INSPIRE].

[27] S. Actis, A. Denner, L. Hofer, J.-N. Lang, A. Scharf and S. Uccirati, RECOLA: REcursive Computation of One-Loop Amplitudes, Comput. Phys. Commun. 214 (2017) 140 [arXiv: 1605.01090] [INSPIRE].

[28] B. Biedermann, S. Bräuer, A. Denner, M. Pellen, S. Schumann and J.M. Thompson, Automation of NLO QCD and EW corrections with Sherpa and Recola, Eur. Phys. J. C 77 (2017) 492 [arXiv : 1704.05783] [INSPIRE].

[29] W. Kilian et al., WHIZARD 2.6, A generic Monte-Carlo integration and event generation package for multi-particle processes, MANUAL, https://whizard.hepforge.org/manual.pdf (2017).

[30] W. Kilian, S. Brass, T. Ohl, J. Reuter, V. Rothe, P. Stienemeier et al., New Developments in WHIZARD Version 2.6, arXiv:1801.08034 [INSPIRE].

[31] A. Denner, S. Dittmaier and L. Hofer, COLLIER - A fortran-library for one-loop integrals, PoS (LL2014) 071 [arXiv: 1407.0087] [INSPIRE].

[32] A. Denner, S. Dittmaier and L. Hofer, Collier: A fortran-based complex one-loop library in extended regularizations, Comput. Phys. Commun. 212 (2017) 220 [arXiv:1604.06792] [INSPIRE].

[33] G. 't Hooft and M.J.G. Veltman, Scalar one loop integrals, Nucl. Phys. B 153 (1979) 365 [INSPIRE].

[34] W. Beenakker and A. Denner, Infrared divergent scalar box integrals with applications in the electroweak standard model, Nucl. Phys. B 338 (1990) 349 [INSPIRE].

[35] S. Dittmaier, Separation of soft and collinear singularities from one loop $N$ point integrals, Nucl. Phys. B 675 (2003) 447 [hep-ph/0308246] [INSPIRE].

[36] A. Denner and S. Dittmaier, Scalar one-loop 4-point integrals, Nucl. Phys. B 844 (2011) 199 [arXiv: 1005.2076] [INSPIRE]. 
[37] G. Passarino and M.J.G. Veltman, One-loop corrections for $e^{+} e^{-}$annihilation into $\mu^{+} \mu^{-}$in the Weinberg model, Nucl. Phys. B 160 (1979) 151 [InSPIRE].

[38] A. Denner and S. Dittmaier, Reduction of one loop tensor five point integrals, Nucl. Phys. B 658 (2003) 175 [hep-ph/0212259] [INSPIRE].

[39] A. Denner and S. Dittmaier, Reduction schemes for one-loop tensor integrals, Nucl. Phys. B 734 (2006) 62 [hep-ph/0509141] [INSPIRE].

[40] A. Denner, S. Dittmaier, M. Roth and L.H. Wieders, Electroweak corrections to charged-current $e^{+} e^{-} \rightarrow 4$ fermion processes: Technical details and further results, Nucl. Phys. B 724 (2005) 247 [Erratum ibid. B 854 (2012) 504] [hep-ph/0505042] [INSPIRE].

[41] S. Catani and M.H. Seymour, A General algorithm for calculating jet cross-sections in NLO QCD, Nucl. Phys. B 485 (1997) 291 [Erratum ibid. B 510 (1998) 503] [hep-ph/9605323] [INSPIRE].

[42] S. Dittmaier, A General approach to photon radiation off fermions, Nucl. Phys. B 565 (2000) 69 [hep-ph/9904440] [INSPIRE].

[43] J. Alwall, R. Frederix, S. Frixione, V. Hirschi, F. Maltoni, O. Mattelaer et al., The automated computation of tree-level and next-to-leading order differential cross sections and their matching to parton shower simulations, JHEP 07 (2014) 079 [arXiv:1405.0301] [INSPIRE].

[44] Z. Nagy and Z. Trócsányi, Next-to-leading order calculation of four jet observables in electron positron annihilation, Phys. Rev. D 59 (1999) 014020 [Erratum ibid. D 62 (2000) 099902] [hep-ph/9806317] [INSPIRE].

[45] A. Denner, J.-N. Lang and S. Uccirati, Recola2: REcursive Computation of One-Loop Amplitudes 2, Comput. Phys. Commun. [arXiv:1711.07388] [INSPIRE].

[46] A. Denner, J.-N. Lang and S. Uccirati, NLO electroweak corrections in extended Higgs Sectors with RECOLA2, JHEP 07 (2017) 087 [arXiv:1705.06053] [INSPIRE].

[47] J.R. Andersen et al., Les Houches 2013: Physics at TeV Colliders: Standard Model Working Group Report, arXiv:1405.1067 [INSPIRE].

[48] A. Buckley, J. Ferrando, S. Lloyd, K. Nordström, B. Page, M. Rüfenacht et al., LHAPDF6: parton density access in the LHC precision era, Eur. Phys. J. C 75 (2015) 132 [arXiv: 1412.7420] [INSPIRE].

[49] NNPDF collaboration, R.D. Ball et al., Parton distributions for the LHC Run II, JHEP 04 (2015) 040 [arXiv: 1410.8849] [INSPIRE].

[50] A. Denner, S. Dittmaier, M. Roth and D. Wackeroth, Electroweak radiative corrections to $e^{+} e^{-} \rightarrow W W \rightarrow 4$ fermions in double pole approximation: The RACOONWW approach, Nucl. Phys. B 587 (2000) 67 [hep-ph/0006307] [INSPIRE].

[51] Particle Data Group collaboration, C. Patrignani et al., Review of Particle Physics, Chin. Phys. C 40 (2016) 100001 [INSPIRE].

[52] LHC Higgs Cross Section Working Group collaboration, D. de Florian et al., Handbook of LHC Higgs Cross Sections: 4. Deciphering the Nature of the Higgs Sector, arXiv:1610.07922 [INSPIRE].

[53] D. Yu. Bardin, A. Leike, T. Riemann and M. Sachwitz, Energy-dependent width effects in $e^{+} e^{-}$-annihilation near the Z-boson pole, Phys. Lett. B 206 (1988) 539 [INSPIRE]. 
[54] L. Basso, S. Dittmaier, A. Huss and L. Oggero, Techniques for the treatment of IR divergences in decay processes at $N L O$ and application to the top-quark decay, Eur. Phys. J. C 76 (2016) 56 [arXiv:1507.04676] [InSPIRE].

[55] M. Cacciari, G.P. Salam and G. Soyez, The anti-k $k_{t}$ jet clustering algorithm, JHEP 04 (2008) 063 [arXiv: 0802.1189] [INSPIRE].

[56] U. Baur, Weak boson emission in hadron collider processes, Phys. Rev. D 75 (2007) 013005 [hep-ph/0611241] [INSPIRE].

[57] A. Bredenstein, A. Denner, S. Dittmaier and S. Pozzorini, NLO QCD corrections to $t \bar{t} b \bar{b}$ production at the LHC: 1. Quark-antiquark annihilation, JHEP 08 (2008) 108 [arXiv: 0807.1248] [INSPIRE].

[58] A. Bredenstein, A. Denner, S. Dittmaier and S. Pozzorini, NLO QCD corrections to $p p \rightarrow t \bar{t} b \bar{b}+X$ at the LHC, Phys. Rev. Lett. 103 (2009) 012002 [arXiv:0905.0110] [INSPIRE].

[59] A. Bredenstein, A. Denner, S. Dittmaier and S. Pozzorini, NLO QCD corrections to t $\bar{t} b \bar{b}$ production at the LHC: 2. Full hadronic results, JHEP 03 (2010) 021 [arXiv:1001.4006] [INSPIRE].

[60] A. Denner, R. Feger and A. Scharf, Irreducible background and interference effects for Higgs-boson production in association with a top-quark pair, JHEP 04 (2015) 008 [arXiv: 1412.5290] [INSPIRE].

[61] S. Catani and B.R. Webber, Infrared safe but infinite: Soft gluon divergences inside the physical region, JHEP 10 (1997) 005 [hep-ph/9710333] [INSPIRE].

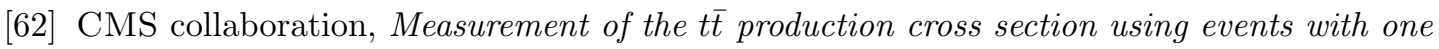
lepton and at least one jet in pp collisions at $\sqrt{s}=13$ TeV, JHEP 09 (2017) 051 [arXiv: 1701.06228] [INSPIRE]. 